\title{
Evolution of danio pigment pattern development
}

\author{
DM Parichy \\ Department of Biology, University of Washington, Box 351800, Seattle, WA 98195, USA
}

\begin{abstract}
Pigment patterns of danio fishes are emerging as a useful system for studying the evolution of developmental mechanisms underlying adult form. Different closely related species within the genera Danio and Devario exhibit a range of pigment patterns including horizontal stripes, vertical bars, and others. In this review, I summarize recent work identifying the genetic and cellular bases for adult pigment pattern formation in the zebrafish Danio rerio,
\end{abstract}

as well as studies of how these mechanisms have evolved in other danios. Together, these analyses highlight the importance of latent precursors at post-embrynoic stages, as well as interactions within and among pigment cell classes, for both pigment pattern development and evolution.

Heredity (2006) 97, 200-210. doi:10.1038/sj.hdy.6800867; published online 12 July 2006

Keywords: pigment pattern; neural crest; melanophore; xanthophore; evolution

\section{Introduction}

Despite tremendous advances in understanding embryogenesis, we still know remarkably little about why organisms look the way they do. How are gene activities orchestrated into the particular shape of a skull bone? How are cell divisions coordinated to sculpt a maple leaf? How does a millipede decide when to stop making segments? Our ignorance is all the more apparent when we seek to understand how differences in form arise between species, or among individuals within a population. Thus, a major remaining goal for developmental and evolutionary biology is to understand the mechanisms responsible for juvenile and adult form, and by extension, how variation in these mechanisms generates corresponding variation in adult form within and among species (Parichy, 2005).

Particularly interesting are the developmental bases for variation in traits that have clear ecological and behavioral significance. Yet, only in a few instances do we know the genetic underpinnings for adaptive differences between populations or closely related species (Abzhanov et al, 2004; Shapiro et al, 2004; Yamamoto et al, 2004; Gompel et al, 2005; Nachman, 2005). In even fewer cases do we know how genetic changes are translated into specific cell behaviors that affect organismal form. One particularly tractable system for addressing these issues is the diversity of color patterns among, and within, species of danio fishes (Figure 1). In this review, I describe some of the developmental and evolutionary insights that have come from recent studies of the genetic and cellular bases for this diversity.

Correspondence: DM Parichy, Department of Biology, University of Washington, Box 351800, Seattle, WA 98195, USA.

E-mail:dparichy@u.washington.edu

Received 10 October 2005; accepted 5 June 2006; published online 12 July 2006

\section{Pigment pattern formation as a system for studying the evolution of developmental mechanisms}

An animal's pigment pattern is one of its most obvious traits, and such patterns have long been of interest to evolutionary biologists because of their ecological and behavioral significance. Spectacular pigment pattern variation is found within every vertebrate class, but the patterns of teleost fishes are among the most tractable for analyzing genetics, cellular behaviors, and ecological and evolutionary mechanisms. Teleost pigment patterns have been implicated in shoaling behavior, mate recognition and mate choice, camouflage and warning coloration, as well as premating isolation during speciation (Endler, 1980; Endler, 1983; Armbruster and Page, 1996; Houde, 1997; Couldridge and Alexander, 2002; Allender et al, 2003; Engeszer et al, 2004).

Besides their interest to evolutionary biologists, pigment patterns have long been a popular system for geneticists, developmental biologists, and mathematical modelers, who have exploited these patterns to study the emergence of biological form (Murray, 1989; Barsh, 1996; Quigley and Parichy, 2002; Dupin and Le Douarin, 2003; Vance and Goding, 2004; Parichy et al, 2006). Not only is this mostly two-dimensional trait easily analyzed even in the living animal, but the cells comprising a pigment pattern are of particular interest because of their neural crest origin. Neural crest cells are a transient embryonic cell type that arises along the dorsal neural tube shortly after neurulation and then disperses throughout the vertebrate embryo. These cells produce a wide variety of derivatives including pigment cells, as well as bone and cartilage of the craniofacial skeleton, neurons and glia of the peripheral nervous system, adrenal chromaffin cells, and others (Hörstadius, 1950; Le Douarin, 1999; Meulemans and Bronner-Fraser, 2004). Normal development of individual neural crest-derived cells requires a complex interplay between factors that are intrinsic (cell autonomous) and extrinsic (cell nonautonomous). Among the 

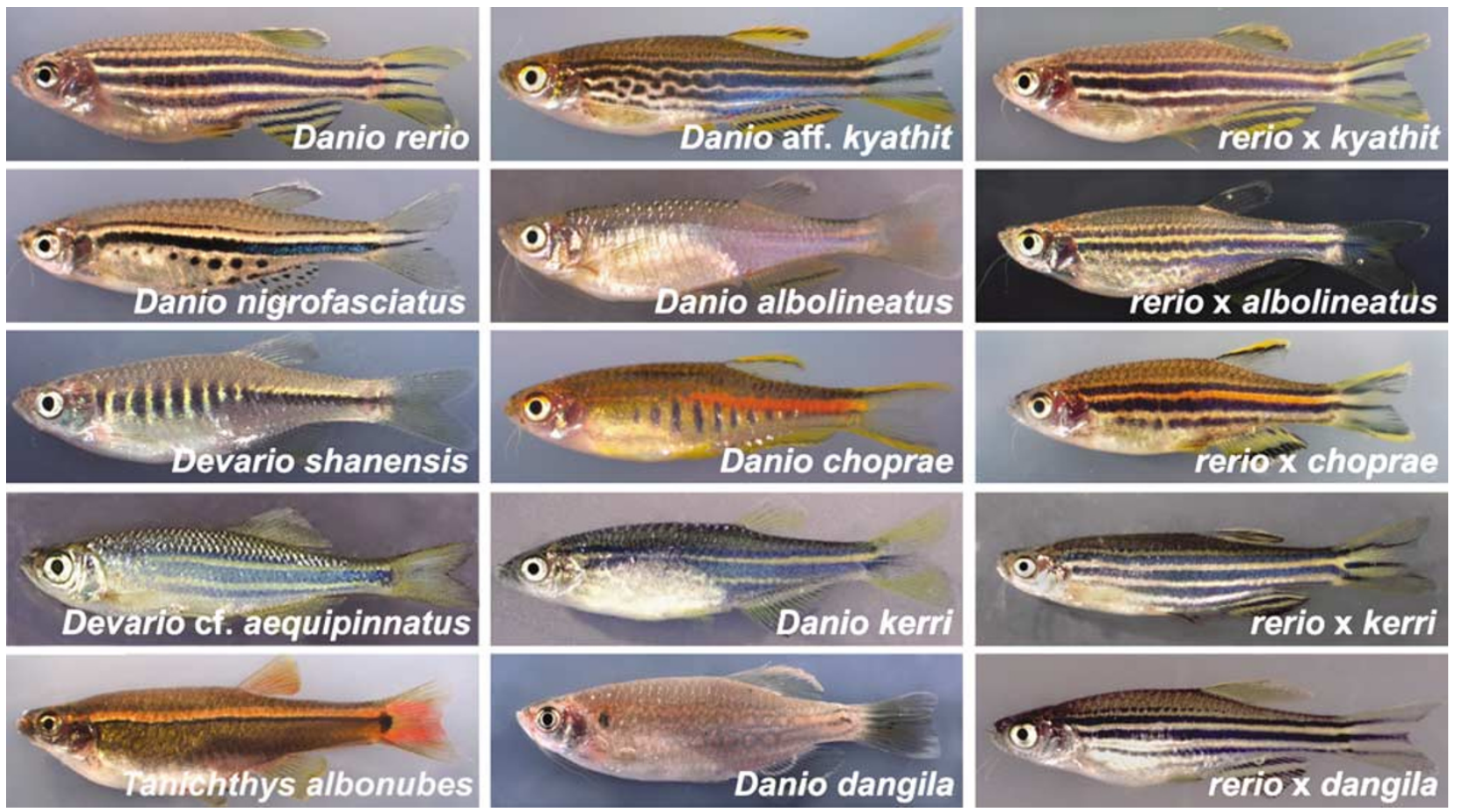

Figure 1 Danio pigment pattern diversity and phenotypes of D. rerio hybrids with other danios. Left and middle columns show phenotypes of several species within Danio, the closely related Devario, and more distant Tanichthys. The group encompasses a range of stripes, bars, uniform, and more complex pigment patterns. Right column shows pigment pattern phenotypes of selected hybrids between $D$. rerio and other danios. Note that patterns typically resemble D. rerio more closely than the heterospecific danio. Species are not all shown at the same scale.

extrinsic factors are a variety of interactions among neural crest-derived cells themselves, and between neural crest-derived cells and other cells they encounter in their extracellular environmnent. Not surprisingly, defects in neural crest development are associated with a diverse array of human disease syndromes (see eg online Mendelian inheritence in man, http://www.ncbi.nlm. nih.gov/entrez/query.fcgi?db=OMIM).

In an evolutionary context, neural crest cells and their derivatives have played major roles in vertebrate diversification (Gans and Northcutt, 1983; Hall, 1999). The interactive nature of neural crest development further suggests that phylogenetic transformations in neural crest derivatives could arise through changes that are intrinsic or extrinsic to neural crest cell lineages. These alternatives have important implications for the degree to which neural crest derivatives evolve independently of other traits, and whether correlated responses to selection can drive the evolution of neural crest derivatives (see below).

Given their developmental and evolutionary importance, and long history of study, pigment patterns are an ideal system for truly integrative approaches linking genetic and cellular mechanisms to their organismal and evolutionary consequences. In this regard, pigment patterns of the zebrafish, Danio rerio, and its relatives are emerging as an especially useful system. D. rerio has become a standard biomedical model organism with a vast array of resources including a whole genome sequence, libraries of expressed sequence tags and large genomic clones, a host of methods for characterizing gene expression and cell behaviors, and a large number of mutant lines (http://zfin.org), particulary affecting the pigment pattern (see below).

For studies of pigment pattern development and evolution, $D$. rerio also is convenient because its relatives have a diverse array of pigment patterns (Fang, 1998; McClure, 1999; Fang and Kottelat, 2000; Quigley and Parichy, 2002; Quigley et al, 2004; Quigley et al, 2005). Figures 1 and 2 show examples of some of these pigment patterns as well as inferred phylogenetic relationships among some of the many species within the group. Whereas $D$. rerio exhibit distinctive horizontal stripes, other species exhibit stripes and spots (D. nigrofasciatus), a few broad stripes (D. kerri), uniform patterns (D. albolineatus), vertical bars (D. choprae), and more complex patterns of stripes and spots (D. dangila). Also shown are several examples of more distantly related species including those within Devario, formerly considered members of Danio (Fang, 2003); for simplicity, fishes of both genera are here referred to as 'danios'. Importantly, most of the techniques and reagents available for $D$. rerio can be applied equally well to these other species.

The arsenal of resources available for $D$. rerio and the diversity and accessibility of other danio patterns makes possible an iterative approach: mechanistic analyses within $D$. rerio can be used to frame hypotheses about how these mechanisms have evolved and these hypotheses can then be tested by experiments on other danios; in turn, experiments at the interspecific level often suggest hypotheses for how patterns develop that can be pursued using $D$. rerio.

The patterns of danio fishes comprise several different classes of pigment cells, or chromatophores, including: black melanophores, yellow or orange xanthophores, red 


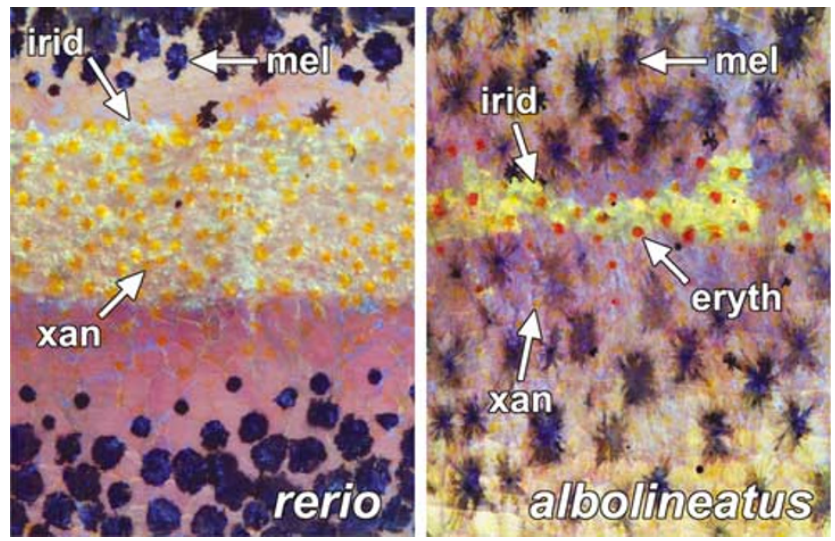

Devario

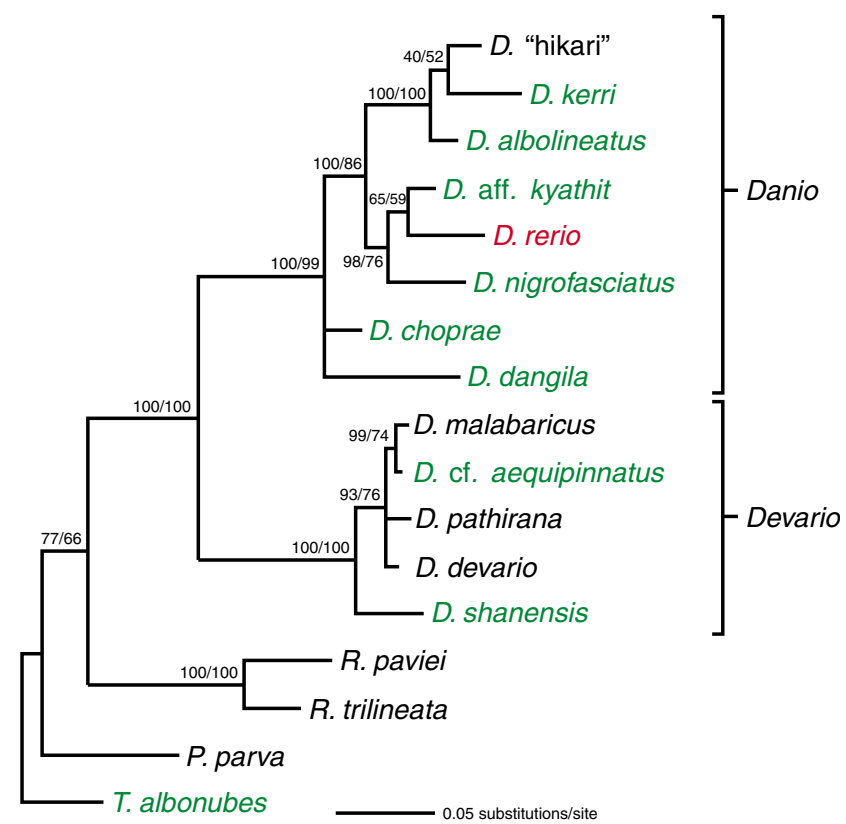

Figure 2 Phylogenetic relationships among danios inferred by maximum likelihood analyses of $12 \mathrm{~S}$ and $16 \mathrm{~S} \mathrm{mtDNA}$. Numbers above branches are support values from Bayesian analysis and nonparametric bootstapping. Many additional danio species are not represented. For details, see Quigley et al (2004). Examples of pigment patterns are shown in Figure 1 for species with names in color.

erythrophores, and iridescent iridophores (Figure 3) (Bagnara, 1998; Hirata et al, 2003; Kelsh, 2004; Parichy et al, 2006). This variety of cell types differs from birds and mammals, which exhibit just a single neural crestderived pigment cell, the melanocyte. Also differing from amniotes is the fate of the pigment itself. Both chromatophores and melanocytes package their pigments within specialized organelles (melanosomes, in the case of melanin), but chromatophores retain their pigments whereas many melanocytes transfer their melanin to developing hair or feathers. Teleost pigment patterns thus reflect the spatial arrangements of the different chromatophore classes themselves, and the pigments serve as cell autonomous markers of their respective cell types. Interestingly, erythrophores are present in some but not all danio species and appear to have arisen or been lost independently across taxa (McClure, 1999; Quigley et al, 2005). The cell lineage relationships among different pigment cell classes are only poorly understood.

\section{Evolution of phenotypes across the metamorphic boundary: the role of post-embryonic stem cells}

Danios resemble many other teleosts in undergoing a distinctive larval-to-adult transformation, or metamorphosis. Indeed, a metamorphosis of one sort or another is present in the majority of animal life cycles, yet the degree to which premetamorphic and postmetamorphic traits depend on common genetic and developmental mechanisms is an important but largely unexplored question: if traits share genes and cell populations across
Figure 3 Pigment cells on the adult flanks of D. rerio (left) and $D$. albolineatus (right). Both species exhibit melanophores (mel), xanthophores (xan), and iridophores (irid). By contrast, only the D. albolineatus pigment pattern includes erythrophores (eryth). Iridophores are distributed widely over the flank of both species but are particularly distinctive within melanophore-free interstripe regions; the intracellular arrangement of iridophore reflecting platelets differs between melanophore stripes and interstripe regions (Hirata et al, 2003). Note the narrower and more irregular interstripe region of $D$. albolineatus as compared to $D$. rerio (whole fish are pictured in Figure 1).

the metamorphic transition, phenotypes appearing at one stage may be biased by the phenotype expressed at another stage, and evolutionary changes in these phenotypes may be limited or enhanced by countervailing or concordant selection operating across stages (Haldane, 1932; Ebenman, 1992; Moran, 1994; Parichy, 1998).

In danios, metamorphosis entails coordinated changes to transform an embryonic or early larval body into that of a juvenile or adult, including: loss of the larval fin fold and development of the adult paired and unpaired fins; changes in the gut and kidneys as well as the peripheral nervous system; increased stratification of the skin; formation of scales; and other changes. The extent of this metamorphosis resembles that seen in some amphibians and is likely to be at least partially under hormonal control (Brown, 1997) (DM Parichy, unpublished data).

The pigment pattern changes dramatically as well (Figure 4a) (Kirschbaum, 1975; Milos and Dingle, 1978; Johnson et al, 1995; Parichy et al, 2000b; Parichy and Turner, 2003b; Kelsh, 2004). Larval D. rerio exhibit stripes of melanophores at the dorsal and ventral margins of the myotomes, along the horizontal myoseptum and lining the yolk mass or abdominal cavity. Iridophores are found within these stripes whereas xanthophores are widely scattered over the flank. By contrast, adult $D$. rerio have 4-6 stripes of melanophores that do not correspond topologically to the earlier larval stripes; xanthophores are abundant between melanophore stripes and iridophores are found across the entire flank. That other danios have larval patterns virtually indistinguishable from $D$. rerio despite their very different adult pigment patterns (Quigley et al, 2004) suggests that pigment patterns are uncoupled both evolutionarily and developmentally across stages.

Either of two models could explain pigment pattern metamorphosis. (1) Adult pigment patterns could arise by remodeling of the early larval pigment pattern: in this 

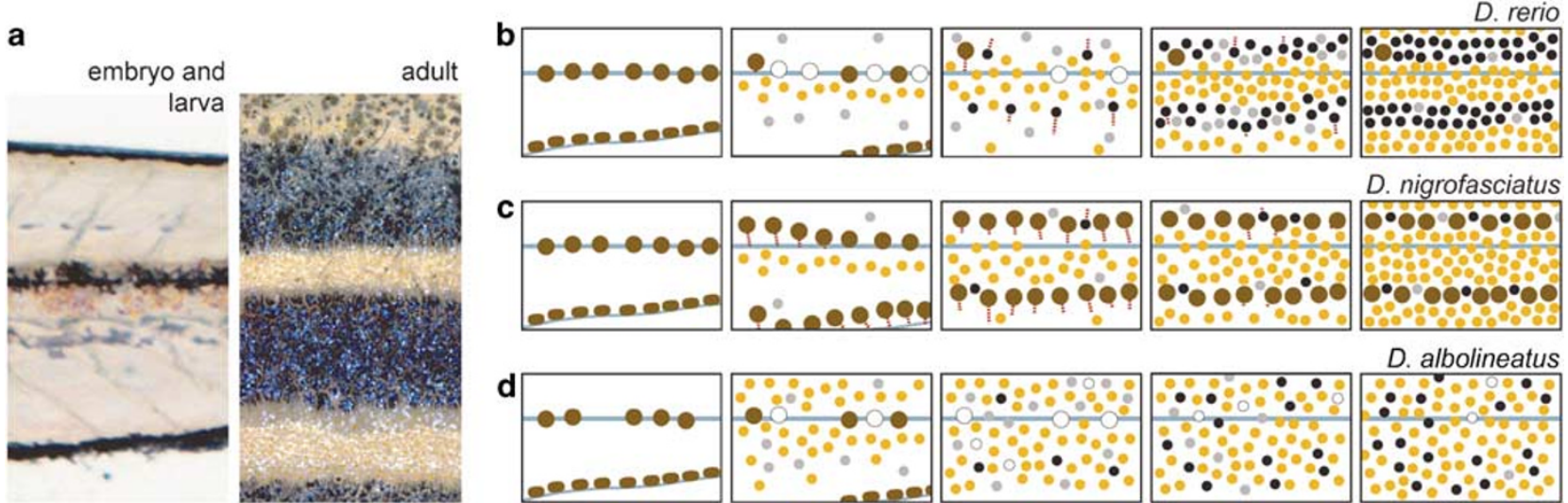

D. nigrofasciatus

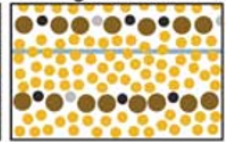

D. albolineatus

Figure 4 Pigment pattern metamorphosis decouples early larval and adult pigment patterns. (a) Close-ups of embryonic/early larval pigment pattern and adult pigment pattern in D. rerio. Images are not to scale (for details, see Parichy and Turner, 2003b). (b, c, d) Schematics illustrating the cellular events of adult pigment pattern formation from the onset of pigment pattern metamorphosis (left panels) to the completion of a juvenile pigment pattern (right panels). (b) In D. rerio, early larval melanophores (large brown cells) are present initially along the horizontal myoseptum (horizontal blue line) and at the margins of the myotomes (the ventral region is illustrated). Subsequently, xanthophores (orange cells) begin to differentiate ventral to the myoseptum and some early larval melanophores are lost (open cells). A few early larval melanophores leave their initial positions to join the developing adult stripes. Metamorphic melanophores (gray cells) differentiate widely scattered over the flank and begin to migrate towards sites of stripe formation (red tracks). These cells become increasingly melanized (small black cells) and additional metamorphic melanophores appear already within the developing adult stripes. (c) In D. nigrofasciatus, early larval melanophores disperse from their initial locations and assume new positions where the adult stripes will form. Some metamorphic melanophores differentiate but these are far fewer than in D. rerio. (d) In D. albolineatus, there are somewhat fewer early larval melanophores along the horizontal myoseptum at the onset of metamorphosis. Many xanthophores differentiate during early metamorphosis and a few melanophores differentiate scattered over the flank. Some of these metamorphic melanophores persist whereas others die (open cells). By late stages of metamorphosis, melanophores and xanthophores are broadly intermingled.

scenario, pigment cells that differentiate in embryos to make the early larval pigment pattern subsequently are reorganized to fashion the adult pigment pattern. (2) Early larval and adult pigment patterns could comprise distinct populations of cells: whereas pigment cells differentiating during embryogenesis would produce the early larval pigment pattern, a different population of pigment cells would differentiate during metamorphosis to produce the adult pattern, with a concomitant loss of the embryonic/early larval population. The ability to observe individual pigment cells throughout development has allowed distinguishing between these two models for the melanophore lineage.

In $D$. rerio, the majority of adult stripe melanophores arise from latent precursors that differentiate during metamorphosis (Parichy and Turner, 2003b; Quigley et al, 2004). At earlier stages, the early larval pigment pattern arises from embryonic melanophores that differentiate directly from neural crest cells; these cells and the pattern they form persist through the early larval period. Beginning about 2 weeks postfertilization, however, new cells start to appear over the flank in between the early larval stripes (Figure $4 \mathrm{~b}$ ). During the next 2 weeks, a new stripe pattern begins to emerge, consisting initially of two adult 'primary' melanophore stripes: one stripe dorsal and one stripe ventral to the original, early larval lateral stripe of melanophores, which runs along the horizontal myoseptum that demarcates the division between dorsal and ventral blocks of muscle. Most early larval melanophores within the lateral stripe are lost during this period, although a few of these cells do succeed in migrating short distances into the developing adult stripes (Figure 2; these changes and other phenotypes described below can be seen in movies available through http://protist.biology.washington. edu/dparichy). Thus, $D$. rerio pigment pattern metamor- phosis is characterized by a segregation of distinct populations of cells, with most of the adult striped pattern comprising newly differentiating metamorphic melanophores and only a small contribution arising from the reorganization of embryonic/early larval melanophores.

That metamorphic melanophores are genetically distinct from embryonic/early larval melanophores is revealed by several $D$. rerio mutants including puma and erbb3 (piccasso) (Figure 5) (Parichy et al, 2003; Parichy and Turner, 2003b; Quigley et al, 2004). Early larval melanophores and the early larval pigment pattern develop normally in these mutants, but during later development, metamorphic melanophores largely fail to appear. Instead, the early larval melanophores persist and move to new locations on the flank, forming a semblance of the normal adult pigment pattern; only during juvenile and adult development do some new 'regulatory' melanophores appear and fill in a partial stripe pattern. These mutants thus seem to decouple pigment pattern metamorphosis from the overall somatic metamorphosis of the fish.

Is the $D$. rerio mode of pigment pattern metamorphosis shared more generally? As a first step in testing this possibility, Quigley et al (2004) examined a D. rerio sister species, D. nigrofasciatus. These fish have fewer stripes, but the stripes that form superficially resemble those of $D$. rerio. Despite this similarity, lineage tracing of individual cells within the adult pigment pattern reveals a very different mode of stripe development: most early larval melanophores persist into the adult and leave their initial positions within the early larval stripes to join the developing adult stripes (Figure 4c). Moreover, far fewer metamorphic melanophores develop during the larvalto-adult transition and molecular analyses reveal that this deficit reflects a dramatically reduced number of 

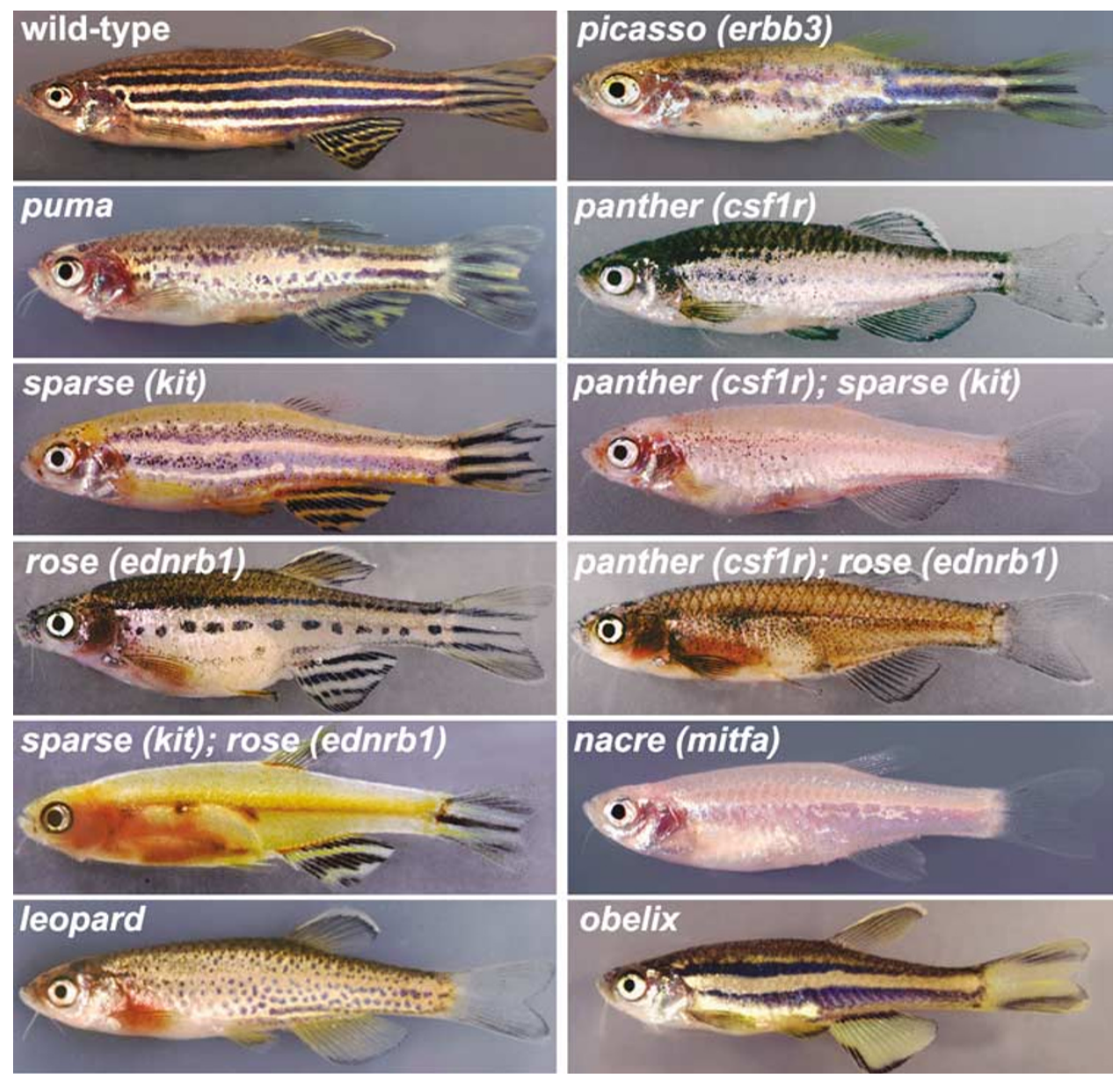

Figure 5 Pigment pattern mutants within D. rerio. Mutant names are shown along with gene identities in parentheses when known. For example, the picasso phenotype results from mutations in errb3 (Lyons et al, 2005; EH Budi and DM Parichy, unpublished data). See text for details.

metamorphic melanophore precursors. Thus, there are at least two very different ways to make a stripe: the $D$. rerio mode that involves differentiation of metamorphic melanophores (from latent precursors) and the $D$. nigrofasciatus mode that involves the redeployment of embryonic/early larval melanophores, with a diminished contribution from metamorphic melanophores.

The mechanisms underlying this interspecific difference in pigment pattern metamorphosis have been analyzed at genetic and cellular levels. The many pigment pattern mutants of $D$. rerio are an important resource for identifying and testing candidate genes and pathways in pigment pattern diversification (Figure 5). Not only do some pigment pattern mutants resemble the naturally occurring patterns of other species, but different species are easily hybridized in the laboratory (Parichy and Johnson, 2001). For pigment pattern mutants of $D$. rerio, this makes possible interspecific complementation tests to examine roles of particular genes or pathways in producing species differences. In standard (intraspecific) complementation tests, two recessive and phenotypically similar mutants are crossed together as a first step in determining if they share a common genetic basis: if the progeny are wild-type, the mutants are inferred to result from lesions in different genes (or pathways); if the progeny exhibit the mutant phenotype, the mutants are inferred to result from lesions in the same gene (or pathway). The same principle can be applied to species differences (Long et al, 1996; Sucena et al, 2003). Hybrids between D. rerio and other danios typically resemble $D$. rerio (Figure 1 ) (Parichy and Johnson, 2001; Quigley et al, 2005); this result is especially dramatic for species with uniform pigment patterns, or vertical bars, in which hybrids instead develop horizontal stripes! Alleles of pigment pattern genes in other species thus are often recessive to those of $D$. rerio, a fact that can be exploited to test whether a gene identified as a $D$. rerio mutant might also differ in its activity between species. If hybrids between the other species and the mutant $D$. rerio resemble hybrids between the other species and wild-type $D$. rerio, there is no evidence for an interspecific difference in the activity of the locus in question; but if the mutant hybrid differs from the wild-type hybrid, this raises the possibility that the same gene or pathway affected in the mutant also differs in its activity between species.

To assess the genetic bases for differences in metamorphic mode between $D$. rerio and D. nigrofasciatus, melanophore lineages were analyzed in hybrids of $D$. nigrofasciatus with several $D$. rerio mutants (Quigley et al, 2004). Mutants that express pigment patterns superficially similar to $D$. nigrofasciatus yielded hybrids that were no different from wild-type controls (reinforcing the notion that pattern and underlying process can be 
very different). By contrast, puma mutant hybrids exhibited dramatically fewer metamorphic melanophores and many more early larval melanophores in the adult pigment pattern than control hybrids. While this result raises the possibility that the puma gene itself has contributed to the interspecific difference, this is unlikely based on genetic mosaic analyses. By transplanting cells between $D$. rerio and $D$. nigrofasciatus, Quigley et al (2004) demonstrated that species differences in metamorphic mode result from evolutionary changes that are extrinsic (nonautonomous) to the melanophore lineages. By contrast, earlier cell transplant studies in $D$. rerio showed that puma acts intrinsically (autonomously) to the melanophore lineages (Parichy et al, 2003). Thus, a more likely explanation for the difference in metamorphic mode lies in evolutionary changes to another gene in the puma pathway. Molecular identification of puma will allow testing this possibility directly, and since hybrids between $D$. rerio and $D$. nigrofasciatus are fertile, quantitative trait locus mapping should help to confirm the number and identity of the genes involved.

Given the alternative modes of pigment pattern metamorphosis found between $D$. rerio and $D$. nigrofasciatus, Quigley et al (2004) extended their analysis to additional species representing the diversity of pigment patterns as well as distinct monophyletic lineages within the group. In each instance, the adult pigment pattern comprised almost entirely metamorphic melanophores with little contribution or persistence of early larval melanophores. These results suggest that a pigment pattern metamorphosis involving the differentiation of metamorphic melanophores and loss of early larval melanophores is ancestral, whereas the reduced metamorphic melanophores and redeployment of early larval melanophores in D. nigrofasciatus represents a unique, derived condition.

More generally, these findings prompt a shift in how one views the evolutionary diversification of pigment patterns. Whereas much of vertebrate evolution clearly depends on changes in the patterning of cells that differentiate directly from neural crest cells during embryogenesis, studies of danio pigment patterns highlight the importance of cells derived from latent precursors long after embryogenesis. Recent studies further suggest that these precursors are stem cells, with self-renewal capability and, in some instances, the capacity to differentiate into multiple lineages (Nishimura et al, 2002; Sieber-Blum et al, 2004) (DM Parichy, unpublished data). A deeper understanding of how pigment patterns develop and evolve will thus require further analyses of these post-embryonic lineages to determine how they are established, maintained, and recruited at particular times and places. It will be interesting to see the extent to which post-embryonic stem cells of presumptive neural crest origin contribute to the development, maintenance, and phylogenetic transformation of other vertebrate traits as well.

\section{Conservation and diversification of gene functions and cell populations: new roles for old friends}

If distinct early larval and metamorphic melanophore populations suggest evolutionary flexibility in how pigment patterns can diversify, additional flexibility is implied by a further partitioning of the metamorphic melanophore populations. Evidence for temporally and genetically separable populations of metamorphic melanophores initially came from studies of $D$. rerio mutants sparse and rose (Figure 5) (Johnson et al, 1995). In wildtype $D$. rerio, melanophore numbers increase steadily during metamorphosis. By contrast, sparse mutant $D$. rerio have fewer melanophores beginning at embryonic stages, and those melanophores that do develop die subsequently, so that by several days post-hatching the larvae completely lack melanophores. During late metamorphosis, however, new melanophores differentiate, already in the positions of adult stripes. A reciprocal melanophore deficit is observed in rose mutants: melanophore numbers are normal from embryonic though early metamorphic stages, but fewer melanophores appear during metamorphosis. The timing of melanophore appearance in sparse and rose mutants raised the possibility of two independent populations: early metamorphic melanophores dependent on sparse, and late metamorphic melanophores dependent on rose. In an elegant test of this model, Johnson et al (1995) predicted that fish doubly mutant for sparse and rose should lack all body melanophores, a prediction confirmed by the fish themselves (Figure 5).

sparse and rose were subsequently identified as zebrafish orthologues of genes long studied for roles in pigment cell development in mammals. sparse corresponds to kit, encoding a receptor tyrosine kinase expressed by the mammalian melanocyte lineage as well as the danio melanophore lineage (Parichy et al, 1999). rose corresponds to endothelin receptor b1 (ednrb1), encoding a seven pass $G$ protein-coupled transmembrane receptor also expressed by these lineages (Parichy et al, 2000a). These findings demonstrated conserved functions for these genes in pigment cell development from mammals to teleosts. Intriguingly, null alleles of mouse Kit and Ednrb completely lack melanocytes and have pleiotropic effects on other cell types (Besmer et al, 1993; Shin et al, 1999; Pla and Larue, 2003; Wehrle-Haller, 2003), whereas in $D$. rerio only embryonic and early appearing metamorphic melanophores require kit, only late appearing metamorphic melanophores require ednrb1, and even null alleles of these genes lack gross pleiotropic defects in other traits. These observations identify fertile ground for investigating how gene functions and pleiotropy itself evolve, particularly as simple gene duplication and partitioning of ancestral functions (Force et al, 1999) seem unlikely to explain genetic requirements for these particular loci (Mellgren and Johnson, 2004; Mellgren and Johnson, 2005).

Double mutant analyses have revealed additional genes required by the kit-independent late metamorphic melanophores in D. rerio. One of these is panther (pfeffer, salz) for which mutants have reduced melanophore numbers (like ednrb1) and also have disrupted stripes and completely lack xanthophores (Figure 5) (Parichy et al, 2000b). The gene responsible for this phenotype encodes the colony-stimulating factor-1 receptor (csf1r, also known as fms). While structurally similar to kit and also previously well studied, mammalian csf1r does not have a role in pigment cell development and instead promotes macrophage and osteoclast development (Dai et al, 2002; Pixley and Stanley, 2004), functions that it 
fulfills in D. rerio as well (Parichy et al, 2000b; Herbomel et al, 2001). Like kit; ednrb1 double mutants, csf1r; kit double mutants lack body melanophores suggesting that ednrb1 and csf1r are both required by the kit-independent melanophore population; consistent with this interpretation, csf1r; ednrb1 double mutants do not have significantly fewer melanophores than csflr mutants, although the cells are arranged differently.

The discovery of temporally and genetically distinct melanophore populations in $D$. rerio raises the question of whether such populations occur in other danios as well. These populations are defined on the basis of mutant phenotypes, so one way to test for their presence is by isolating the corresponding mutants in other species. This approach was applied recently to $D$. albolineatus, in which an adult stripe pattern has been lost evolutionarily (Figure 1, 4d). This species has far fewer melanophores than $D$. rerio particularly during late stages of metamorphosis, when the kit-independent melanophores develop in D. rerio (Quigley et al, 2005). Moreover, interspecific complementation tests identified csf1r or its pathway as candidates for contributing to this difference in melanophore number and patterning between species (Parichy and Johnson, 2001). Together, these observations suggest that $D$. albolineatus might lack the late-appearing, kit-independent, csflr-dependent melanophore population that normally differentiates in a stripe pattern in $D$. rerio. If kit-independent melanophores already have been lost evolutionarily, then kit mutant $D$. albolineatus should lack all body melanophores (and so should resemble csf1r; kit double mutant $D$. rerio, Figure 5). Yet, a simple genetic experiment disproves this hypothesis. Noncomplementation screening of mutagenized $D$. albolineatus families against kit mutant $D$. rerio allowed the isolation of a kit mutant in the D. albolineatus background (RJ Nuckels and DM Parichy, unpublished data). Like kit mutant $D$. rerio, kit mutant $D$. albolineatus have fewer melanophores as embryos, completely lack melanophores by early larval stages and through early metamorphosis, then ultimately recover melanophores during late metamorphosis. Thus, complete loss of a kitindependent melanophore population cannot be responsible for the reduced melanophore number, or loss of stripes, in $D$. albolineatus. These results demonstrate at least some conservation of metamorphic melanophore populations between $D$. rerio and $D$. albolineatus despite their radically different pigment patterns. As corresponding mutants of $D$. rerio pigment pattern genes are identified in other danios and more distant teleosts, such as the medaka Oryzias latipes (Kelsh et al, 2004; Lynn Lamoreux et al, 2005), it will be interesting to see the extent to which gene functions and dependent melanophore populations resemble those of $D$. rerio.

\section{Pattern-forming mechanisms and their phenotypic outcomes: cooperation and the evolution of noncooperation in cell behaviors}

If different classes of metamorphic melanophores and other pigment cells provide the raw material for adult pigment pattern diversification, how are the different pigment patterns themselves produced? Answering this question requires not merely identifying genes that contribute to species or population differences, but also elucidating how differences in gene activity are translated through cellular processes - differentiation and morphogenesis - into differences in form. The many pigment pattern mutants of $D$. rerio are a useful starting point for dissecting these mechanisms, and a few of these have already proven helpful in defining the cellular context for interpreting species differences.

Studies of csf1r mutant $D$. rerio reveal a critical role for cellular interactions in stripe development. While csf1r mutant $D$. rerio has fewer melanophores than wild-type, the failure of normal stripe development suggested additional roles for this gene in organizing melanophores. In wild-type larvae, initially dispersed melanophores migrate into stripes during adult pigment pattern formation as other melanophores differentiate already in the final positions of the stripes. In csflr mutant larvae, melanophores fail to migrate in a directed manner, these cells die at inappropriate locations on the flank, and the late-appearing metamorphic melanophores fail to differentiate at sites of stripe formation (Parichy et al, 2000b). An obvious - and incorrect - interpretation would be that csf1r directly controls melanophore behaviors, a notion that is suspect in no small part because melanophores do not themselves express detectable levels of csf1r. Instead, csf1r acts through the xanthophore lineage. These cells that express csf1r are absent in csf1r mutants, and genetic mosaic analyses demonstrate the role of these cells in organizing melanophores (Parichy and Turner, 2003a). One such experiment used nacre mutant zebrafish as a source of donor cells for making chimeric adults (Figure 5). nacre mutants have a mutation in microphthalmia-a (Lister et al, 1999), which encodes a transcription factor essential within the melanophore lineage for the specification and differentiation of these cells (Goding, 2000; Steingrimsson et al, 2004); thus nacre mutants completely lack melanophores, although they do develop xanthophores. By transplanting cells from nacre mutants (melanophore ${ }^{-}$, xanthophore ${ }^{+}$) to csf1r mutants (melanophore ${ }^{+}$, xanthophore ${ }^{-}$), it was possible to reintroduce the two cell populations and ask if stripes could be recovered (Figure 6). In chimeras that failed to develop donor xanthophores, normal stripes were absent; but in chimeras that developed donor xanthophores, melanophores in the vicinity of these cells formed a stripe pattern. These results were subsequently confirmed by independent studies (Maderspacher and Nusslein-Volhard, 2003) and together suggest that interactions between melanophores and csf1r-dependent cells of the xanthophore lineage are needed for $D$. rerio stripe development.

Interactions between melanophores and the xanthophore lineage are required not only for stripe formation but also for stripe maintenance. This was demonstrated with an approach that is relatively straightforward in $D$. rerio, the isolation and analysis of temperature-sensitive alleles. Conditional, and particularly temperature-sensitive, mutant alleles have long been used to identify critical periods for gene function. Such alleles also can be especially valuable when a gene has early essential functions, such that embryonic lethality of mutants would otherwise preclude analyzing potential functions during later development. The ability to grow $D$. rerio at a range of temperatures means that one can readily isolate temperature-sensitive alleles, either by incorporating a temperature shift into an initial phenotype-based 

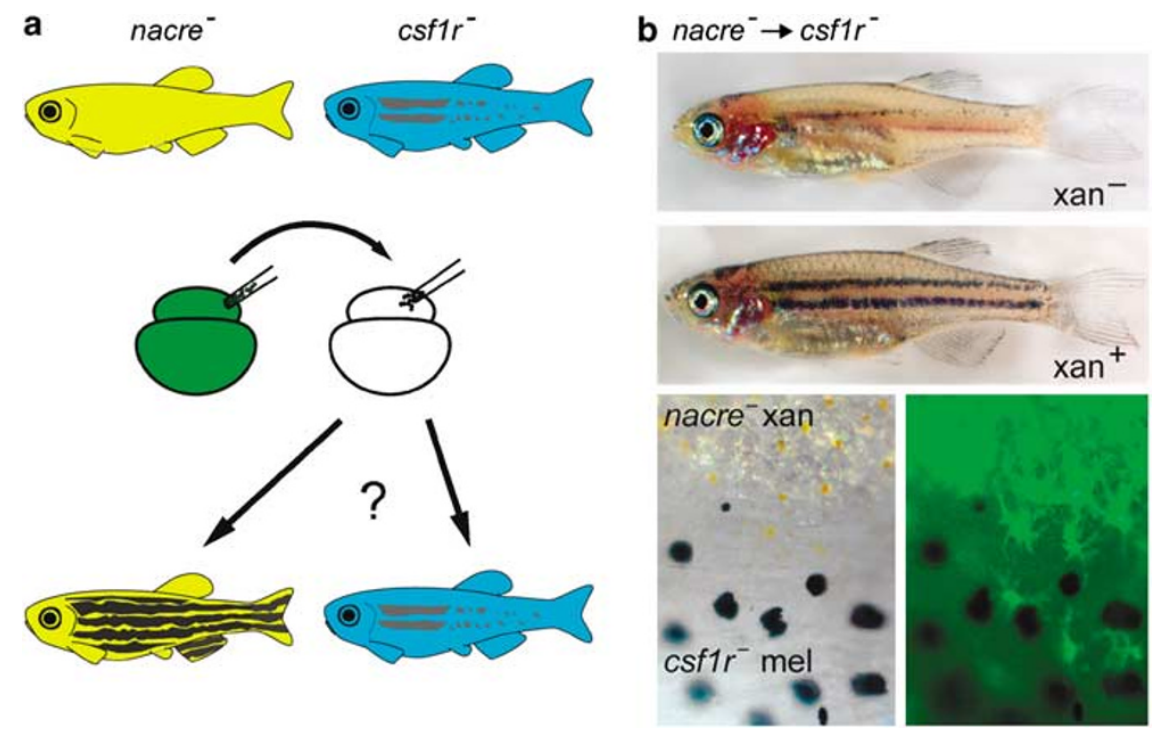

Figure 6 Interactions between melanophores and cells of the xanthophore lineage contribute to D. rerio stripe formation (a) Transplants between nacre mutant and csf1r mutant $D$. rerio allow testing whether juxtaposition of xanthophores and melanophores are sufficient for stripe development. (b) Phenotypes of genetic mosaic adults. When donor-derived xanthophore fail to develop, a typical csf1r mutant pigment pattern results. When donor-derived xanthophores are present, however, stripes are rescued. Lower panels show details of these stripes with donor nacre mutant xanthophores (identified by a ubiquitously expressed GFP) adjacent to host csf1r mutant melanophores.

screen (Johnson and Weston, 1995; Parichy and Turner, $2003 \mathrm{~b})$, or by noncomplementation screening at a different temperature for new alleles of an existing mutant locus (Rawls and Johnson, 2003). The latter approach was used to find temperature-sensitive csflr alleles: $\sim 75$ new alleles were produced and of $\sim 42$ tested for temperature sensitivity, three strong temperature-sensitive alleles were identified (Parichy and Turner, 2003a). When these mutants are reared at a permissive temperature $\left(24^{\circ} \mathrm{C}, \mathrm{csf} 1 \mathrm{r}\right.$ 'on'), they develop a wild-type complement of xanthophores and wild-type adult stripes. When reared at a restrictive temperature $\left(33^{\circ} \mathrm{C}\right.$, csf1r 'off'), they completely lack xanthophores and fail to form organized stripes. By shifting fish reciprocally between permissive and restrictive temperatures at a range of stages from embryo to adult, it was shown that ablating csf1r activity at any time resulted in the loss of xanthophores and degeneration of adult melanophore stripes, and conversely, activating csf1r at any time allowed xanthophore recruitment and the concomitant organization of adult melanophore stripes. Thus, interactions between melanophores and csf1r-dependent cells of the xanthophore lineage are required for initial stripe formation and these interactions continue to be required for stripe maintenance even in the adult.

Two other $D$. rerio mutants identify additional genes believed to be involved in pigment cell interactions: leopard and obelix (jaguar) (Figure 5) (Rawls et al, 2001; Maderspacher and Nusslein-Volhard, 2003). The original leopard mutant allele develops melanophore spots instead of stripes but an allelic series reveals defects ranging in severity from broken melanophore stripes to nearly uniformly dispersed melanophores and xanthophores (Asai et al, 1999). obelix mutants develop a striped pattern but this pattern is defective in at least two ways. First, obelix mutants develop fewer, broader stripes. Whereas wild-type zebrafish develop two adult primary stripes, with additional 'secondary' stripes added dorsally and ventrally as the fish grow, obelix mutants fail to normally curtail the dorsal and ventral boundaries of the primary stripes, resulting in two abnormally wide stripes, and then fail to add more stripes. Second, numerous xanthophores develop even within the melanophore stripes that do form. Both mutant phenotypes have been interpreted to reflect underlying defects in boundary formation. Cell transplants and epistasis analyses have suggested a model in which leopard promotes attractive homotypic interactions (melanophore-melanophore, xanthophore-xanthophore), as well as both attractive and repulsive heterotypic interactions (melanophore-xanthophore); obelix instead promotes only attractive homotypic interactions (melanophoremelanophore) (Maderspacher and Nusslein-Volhard, 2003).

The molecular natures of interactions within and among pigment cell classes have yet to be revealed. A wide variety of mechanisms could be involved, including direct contacts between melanophores, xanthophores, or their precursors, as well as indirect mechanisms involving secreted signaling molecules, trophic factors, or even intermediary cell types. Interactions within and among pigment cell classes were also inferred long ago from studies of developing and regenerating danio fins (Goodrich and Nichols, 1931; Goodrich et al, 1954; Goodrich and Greene, 1959), and more recently, from studies of stripe and bar development in salamander larvae (Epperlein and Lofberg, 1990; Parichy, 1996; Parichy, 2001), suggesting that such mechanisms may be relatively widespread.

Pattern-generating mechanisms that depend on interactions among pigment cells could be especially significant evolutionarily, if such mechanisms allow patterns to develop in a 'modular' way, largely independent of other traits (Maynard Smith et al, 1985; von Dassow and Munro, 1999; Beldade et al, 2002). New variants could thus arise, and so be available 
for selection, without correlated alterations in other characters. For instance, a single pattern-generating mechanism, such as interactions between melanophores and the xanthophore lineage, could produce a variety of patterns depending on when and where it is deployed. Empirical support for this notion comes from the caudal fins of temperature-sensitive csflr mutants (Parichy and Turner, 2003a). In wild-type, the fin exhibits horizontal stripes; but when csf1r is first activated only in adults, fin stripes with haphazard orientations develop. This suggests that cues required normally to orient fin stripes are not present, or not recognized, at these later stages. Thus, it is conceivable - although by no means proven - that horizontal stripes in species like $D$. rerio and vertical bars in species like $D e$. shanensis simply reflect differences in the timing of otherwise conserved interactions among pigment cells, and in the specific cues for orientation present during those interactions. Consistent with this notion, vertical bars develop much later than horizontal stripes (DM Parichy, unpublished data). These considerations suggest a model by which evolutionary changes in pigment pattern often proceed without correlated changes in other characters, although patterns may be biased in particular ways by molecular and cellular features of the extracellular environment encountered by developing pigment cells (Parichy, 1996). Important goals for future studies will be to identify the mechanisms of interactions within and among pigment cell classes, and the types of interactions between pigment cells and their extracellular environment.

The discovery that pigment cell interactions contribute to stripe formation also casts a new light on stripe loss in D. albolineatus. Unlike hybrids between wild-type $D$. rerio and D. albolineatus, which develop stripes, hybrids between csf1r mutant $D$. rerio and $D$. albolineatus lack organized stripes (Parichy and Johnson, 2001; Quigley et al, 2005). This observation and the csf1r mutant $D$. rerio phenotype suggest three models for the evolution of stripe loss in D. albolineatus. (1) D. albolineatus have lost the late-appearing, kit-independent population of metamorphic melanophores that develops already in the position of adult stripes in $D$. rerio. The phenotype of kit mutant D. albolineatus (above) shows this is not true. (2) $D$. albolineatus have lost the csf1r-dependent xanthophore lineage and so these cells are not present to exert their effects on melanophores. Also untrue: D. albolineatus actually have many more xanthophores and their precursors than D. rerio (Quigley et al, 2005). (3) Melanophore behaviors are themselves altered, perhaps owing to changes in how these cells interact with one another or with cells of the xanthophore lineage. Support for this last model comes from the absence of directed melanophore migration and increased frequency of melanophore death in D. albolineatus, reminiscent of $c s f 1 r$ mutant $D$. rerio, as well as from the intermingling of pigment cell classes in D. albolineatus, resembling obelix and some leopard mutant $D$. rerio. Phenotypes of interspecific hybrids between $D$. albolineatus and other $D$. rerio mutants also are consistent with this model. These and other experiments further suggest that the csf1r-dependence of hybrid pigment patterns reflects an evolutionarily increased sensitivity of $D$. albolineatus xanthophore development to changes in csf1r activity, or evolutionary changes to molecular interactions within the csflr pathway itself (for details, see Quigley et al, 2005).

\section{Conclusions}

Studies of danio pigment patterns are beginning to reveal basic mechanisms of pigment cell differentiation and morphogenesis during the development of adult form, and how changes in these mechanisms can generate pattern variation within and among species. Together, these studies suggest a model for the development and evolution of pigment patterns within this group (Figure 7).

Some significant challenges remain. Of the developmental questions, some of the most interesting concern the mechanisms of pigment cell interactions, the cues employed for orienting patterns, potential correlations between pigment patterns and somatic growth (McClure and McCune, 2003), and how stem cells are called upon to make post-embryonic lineages. Of the evolutionary questions, it will be especially interesting to assess the type and magnitude of population variation in pigment pattern and alleles at pigment pattern loci, as well as how specific pigment patterns fit into the natural histories of different danio species. Clearly, such challenges also provide outstanding opportunities for integrative studies that will bridge the gaps between genes

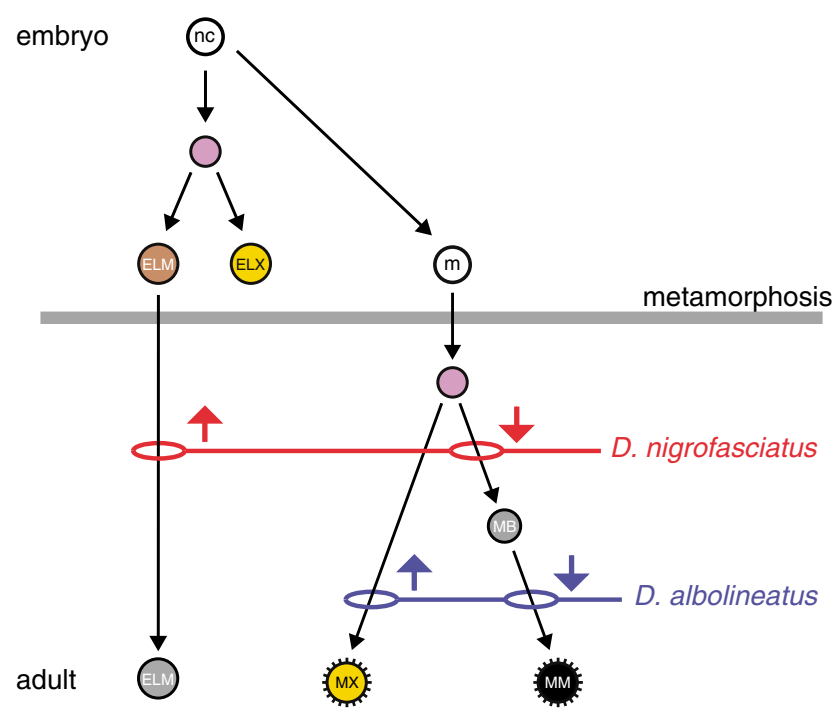

Figure 7 Model for evolutionary changes in danio pigment cell lineages. In embryos, neural crest cells differentiate into a diverse array of cell types including early larval melanophores (ELM) and early larval xanthophores (ELX). Additional cells are hypothesized to serve as stem cells $(m)$ that can be recruited to differentiate during metamorphosis. During the larval-to-adult transformation of $D$. rerio, relatively few early larval melanophores persist into adult stripes and instead numerous metamorphic melanophores (MM) and metamorphic xanthophores (MX) are recruited. Interactions within and among pigment cell classes contribute to stripe formation (schematized by hatch marks on MX and MM), as do other presently unknown cues required for setting positions and orientations of stripes. In D. nigrofasciatus, more ELM persist and fewer MM develop owing to an early block in the metamorphic melanophore lineages that also affects undifferentiated melanoblasts (MB). In D. albolineatus, there are more xanthophores and fewer metamorphic melanophores than in D. rerio, as well as hypothesized differences in pigment cell interactions. 
and phenotypes, individuals and populations, and populations and species.

\section{Acknowledgements}

Unpublished results presented here were supported by NIH R01 GM62812 and NSF IOB-044517. I thank MK Iovine and A Brand for their hospitality while writing this review and $\mathrm{M}$ Mills for comments on the manuscript.

\section{References}

Abzhanov A, Protas M, Grant BR, Grant PR, Tabin CJ (2004). Bmp4 and morphological variation of beaks in Darwin's finches. Science 305: 1462-1465.

Allender CJ, Seehausen O, Knight ME, Turner GF, Maclean N (2003). Divergent selection during speciation of Lake Malawi cichlid fishes inferred from parallel radiations in nuptial coloration. Proc Natl Acad Sci USA 100: 14074-14079.

Armbruster JW, Page LM (1996). Convergence of a cryptic saddle pattern in benthic freshwater fishes. Environ Biol Fishes 45: 249-257.

Asai R, Taguchi E, Kume Y, Saito M, Kondo S (1999). Zebrafish leopard gene as a component of the putative reactiondiffusion system. Mech Dev 89: 87-92.

Bagnara JT (1998). Chapter 2. Comparative anatomy and physiology of pigment cells in nonmammalian tissues. In: Nordland JJ, Boissy RE, Hearing VJ, King RA, Ortonne JP (eds) The Pigmentary System and Its Disorders, Ist edn. Oxford University Press: New York, NY. pp 9-40.

Barsh GS (1996). The genetics of pigmentation: from fancy genes to complex traits. Trends Genet 12: 299-305.

Beldade P, Koops K, Brakefield PM (2002). Modularity, individuality, and evo-devo in butterfly wings. Proc Natl Acad Sci USA 99: 14262-14267.

Besmer P, Manova K, Duttlinger R, Huang EJ, Packer A, Gyssler $\mathrm{C}$ et al (1993). The kit-ligand (steel factor) and its receptor c-kit/W: pleiotropic roles in gametogenesis and melanogenesis. Dev Suppl 1993: 125-137.

Brown DD (1997). The role of thyroid hormone in zebrafish and axolotl development. Proc Natl Acad Sci USA 94: 13011-13016.

Couldridge VCK, Alexander GJ (2002). Color patterns and species recognition in four closely related species of Lake Malawi cichlid. Behav Ecol 13: 59-64.

Dai XM, Ryan GR, Hapel AJ, Dominguez MG, Russell RG, Kapp S et al (2002). Targeted disruption of the mouse colonystimulating factor 1 receptor gene results in osteopetrosis, mononuclear phagocyte deficiency, increased primitive progenitor cell frequencies, and reproductive defects. Blood 99: 111-120.

Dupin E, Le Douarin NM (2003). Development of melanocyte precursors from the vertebrate neural crest. Oncogene 22: 3016-3023.

Ebenman B (1992). Evolution in organisms that change their niches during the life-cycle. Am Natur 139: 990-1021.

Endler JA (1980). Natural-selection on color patterns in poeciliareticulata. Evolution 34: 76-91.

Endler JA (1983). Natural and sexual selection on color patterns in Poeciliid fishes. Env Biol Fishes 9: 173-190.

Engeszer RE, Ryan MJ, Parichy DM (2004). Learned social preference in zebrafish. Curr Biol 14: 881-884.

Epperlein HH, Lofberg J (1990). The development of the larval pigment patterns in Triturus alpestris and Ambystoma mexicanum. Adv Anat Embryol Cell Biol 118: 1-99.

Fang F (1998). Danio kyathit, a new species of cyprinid fish from Myitkyina, northern Myanmar. Ichthyol Explor Freshwaters 8: 273-280.
Fang F (2003). Phylogenetic analysis of the Asian cyprinid genus Danio (Teleostei, Cyprinidae). Copeia 2003: 714-728.

Fang F, Kottelat M (2000). Danio roseus, a new species from the Mekong basin in northeastern Thailand and northwestern Laos (Teleostei: Cyprinidae). Ichthyol Explor Freshwaters 11: 149-154.

Force A, Lynch M, Pickett FB, Amores A, Yan YL, Postlethwait J (1999). Preservation of duplicate genes by complementary, degenerative mutations. Genetics 151: 1531-1545.

Gans C, Northcutt RG (1983). Neural crest and the origin of vertebrates: a new head. Science 220: 268-274.

Goding CR (2000). Mitf from neural crest to melanoma: signal transduction and transcription in the melanocyte lineage. Genes Dev 14: 1712-1728.

Gompel N, Prud'homme B, Wittkopp PJ, Kassner VA, Carroll SB (2005). Chance caught on the wing: cis-regulatory evolution and the origin of pigment patterns in Drosophila. Nature 433: 481-487.

Goodrich HB, Greene JM (1959). An experimental analysis of the development of a color pattern in the fish Brachydanio albolineatus Blyth. J exp Zool 141: 15-45.

Goodrich HB, Marzullo CM, Bronson WR (1954). An analysis of the formation of color patterns in two fresh-water fish. J Exp Zool 125: 487-505.

Goodrich HB, Nichols R (1931). The development and the regeneration of the color pattern in Brachydanio rerio. J Morphol 52: 513-523.

Haldane JBS (1932). The time of action of genes, and its bearing on some evolutionary problems. Am Natur 66: 5-24.

Hall BK (1999). The Neural Crest in Development and Evolution. Springer-Verlag: New York, NY.

Herbomel P, Thisse B, Thisse C (2001). Zebrafish early macrophages colonize cephalic mesenchyme and developing brain, retina, and epidermis through a M-CSF receptor-dependent invasive process. Dev Biol 238: 274-288.

Hirata M, Nakamura K, Kanemaru T, Shibata Y, Kondo S (2003). Pigment cell organization in the hypodermis of zebrafish. Dev Dyn 227: 497-503.

Houde AE (1997). Sex, Color, and Mate Choice in Guppies. Princeton University Press: Princeton, NJ.

Hörstadius S (1950). The Neural Crest: its Properties and Derivatives in Light of Experimental Research. Oxford University Press: London, England.

Johnson SL, Africa D, Walker C, Weston JA (1995). Genetic control of adult pigment stripe development in zebrafish. Dev Biol 167: 27-33.

Johnson SL, Weston JA (1995). Temperature-sensitive mutations that cause stage-specific defects in Zebrafish fin regeneration. Genetics 141: 1583-1595.

Kelsh RN (2004). Genetics and evolution of pigment patterns in fish. Pigment Cell Res 17: 326-336.

Kelsh RN, Inoue C, Momoi A, Kondoh H, Furutani-Seiki M, Ozato $\mathrm{K}$ et al (2004). The Tomita collection of medaka pigmentation mutants as a resource for understanding neural crest cell development. Mech Dev 121: 841-859.

Kirschbaum F (1975). Untersuchungen über das Farbmuster der Zebrabarbe Brachydanio rerio (Cyprinidae, Teleostei). Wilhelm Roux's Arch 177: 129-152.

Le Douarin NM (1999). The Neural Crest. Cambride University Press: Cambridge.

Lister JA, Robertson CP, Lepage T, Johnson SL, Raible DW (1999). Nacre encodes a zebrafish microphthalmia-related protein that regulates neural-crest-derived pigment cell fate. Development 126: 3757-3767.

Long AD, Mullaney SL, Mackay TFC, Langley CH (1996). Genetic interactions between naturally occurring alleles at quantitative trait loci and mutant alleles at candidate loci affecting bristle number in Drosophila melanogaster. Genetics 144: $1497-1510$. 
Lynn Lamoreux M, Kelsh RN, Wakamatsu Y, Ozato K (2005). Pigment pattern formation in the medaka embryo. Pigment Cell Res 18: 64-73.

Lyons DA, Pogoda HM, Voas MG, Woods IG, Diamond B, Nix R et al (2005). erbb3 and erbb2 are essential for Schwann cell migration and myelination in zebrafish. Curr Biol 15: 513-524

Maderspacher F, Nusslein-Volhard C (2003). Formation of the adult pigment pattern in zebrafish requires leopard and obelix dependent cell interactions. Development 130: 34473457.

Maynard Smith J, Burian R, Kauffman S, Alberch P, Campbell J, Goodwin B et al (1985). Developmental constraints and evolution. Q Rev Biol 60: 265-287.

McClure M (1999). Development and evolution of melanophore patterns in fishes of the genus Danio (Teleostei: Cyprinidae). J Morphol 241: 83-105.

McClure M, McCune AR (2003). Evidence for developmental linkage of pigment patterns with body size and shape in danios (Teleostei: Cyprinidae). Evol Int J Org Evol 57: 18631875.

Mellgren EM, Johnson SL (2004). A requirement for kit in embryonic zebrafish melanocyte differentiation is revealed by melanoblast delay. Dev Genes Evol 214: 493-502.

Mellgren EM, Johnson SL (2005). kitb, a second zebrafish ortholog of mouse kit. Dev Genes Evol 215: 1-8.

Meulemans D, Bronner-Fraser M (2004). Gene-regulatory interactions in neural crest evolution and development. Dev Cell 7: 291-299.

Milos N, Dingle AD (1978). Dynamics of pigment pattern formation in the zebrafish, Brachydanio rerio. I. Establishment and regulation of the lateral line melanophore stripe during the first eight days of development. J Exp Zool 205: 205-216.

Moran NA (1994). Adaptation and constraint in the complex life-cycles of animals. Ann Rev Ecol Syst 25: 573-600.

Murray JD (1989). Math Biol. Springer-Verlag: Berlin.

Nachman MW (2005). The genetic basis of adaptation: lessons from concealing coloration in pocket mice. Genetica 123: 125-136.

Nishimura EK, Jordan SA, Oshima H, Yoshida H, Osawa M, Moriyama $M$ et al (2002). Dominant role of the niche in melanocyte stem-cell fate determination. Nature 416: 854-860.

Parichy DM (1996). Pigment patterns of larval salamanders (Ambystomatidae, Salamandridae): the role of the lateral line sensory system and the evolution of pattern-forming mechanisms. Dev Biol 175: 265-282.

Parichy DM (1998). Experimental analysis of character coupling across a complex life cycle: pigment pattern metamorphosis in the tiger salamander, Ambystoma tigrinum tigrinum. J Morphol 237: 53-67.

Parichy DM (2001). Chapter 7. Pigment patterns of ectothermic vertebrates: heterochronic vs non-heterochronic models for pigment pattern evolution. In: Zelditch ML (ed) Beyond Heterochrony. John Wiley \& Son, Inc.: New York. pp 229-269.

Parichy DM (2005). Chapter 20. Variation and developmental biology: prospects for the future. In: Halgrimsson B, Hall BK (eds) Variation: a Hierarchical Examination of a Central Concept in Biology. Academic Press: New York, NY. pp 475-498.

Parichy DM, Johnson SL (2001). Zebrafish hybrids suggest genetic mechanisms for pigment pattern diversification in Danio. Dev Genes Evol 211: 319-328.

Parichy DM, Mellgren EM, Rawls JF, Lopes SS, Kelsh RN, Johnson SL (2000a). Mutational analysis of endothelin receptor b1 (rose) during neural crest and pigment pattern development in the zebrafish Danio rerio. Dev Biol 227 294-306.

Parichy DM, Ransom DG, Paw B, Zon LI, Johnson SL (2000b). An orthologue of the kit-related gene fms is required for development of neural crest-derived xanthophores and a subpopulation of adult melanocytes in the zebrafish, Danio rerio. Development 127: 3031-3044.
Parichy DM, Rawls JF, Pratt SJ, Whitfield TT, Johnson SL (1999). Zebrafish sparse corresponds to an orthologue of c-kit and is required for the morphogenesis of a subpopulation of melanocytes, but is not essential for hematopoiesis or primordial germ cell development. Development 126: 34253436.

Parichy DM, Reedy MV, Erickson CA (2006). Chapter 5. Regulation of melanoblast migration and differentiation. In: Nordlund JJ, Boissy RE, Hearing VJ, King RA, Ortonne JP (eds) The Pigmentary System and its Disorders, 2nd edn. Oxford University Press: New York, NY, pp 108-139.

Parichy DM, Turner JM (2003a). Temporal and cellular requirements for Fms signaling during zebrafish adult pigment pattern development. Development 130: 817-833.

Parichy DM, Turner JM (2003b). Zebrafish puma mutant decouples pigment pattern and somatic metamorphosis. Dev Biol 256: 242-257.

Parichy DM, Turner JM, Parker NB (2003). Essential role for puma in development of postembryonic neural crest-derived cell lineages in zebrafish. Dev Biol 256: 221-241.

Pixley FJ, Stanley ER (2004). CSF-1 regulation of the wandering macrophage: complexity in action. Trends Cell Biol 14: 628638.

Pla P, Larue L (2003). Involvement of endothelin receptors in normal and pathological development of neural crest cells. Int J Dev Biol 47: 315-325.

Quigley IK, Manuel JL, Roberts RA, Nuckels RJ, Herrington ER, Macdonald EL et al (2005). Evolutionary diversification of pigment pattern in Danio fishes: differential fms dependence and stripe loss in D. albolineatus. Development 132: 89-104.

Quigley IK, Parichy DM (2002). Pigment pattern formation in zebrafish: a model for developmental genetics and the evolution of form. Microsc Res Tech 58: 442-455.

Quigley IK, Turner JM, Nuckels RJ, Manuel JL, Budi EH, Macdonald EL et al (2004). Pigment pattern evolution by differential deployment of neural crest and post-embryonic melanophore lineages in Danio fishes. Development 131: 60536069.

Rawls JF, Johnson SL (2003). Temporal and molecular separation of the kit receptor tyrosine kinase's roles in zebrafish melanocyte migration and survival. Dev Biol 262: 152-161.

Rawls JF, Mellgren EM, Johnson SL (2001). How the zebrafish gets its stripes. Dev Biol 240: 301-314.

Shapiro MD, Marks ME, Peichel CL, Blackman BK, Nereng KS, Jonsson B et al (2004). Genetic and developmental basis of evolutionary pelvic reduction in threespine sticklebacks. Nature 428: 717-723.

Shin MK, Levorse JM, Ingram RS, Tilghman SM (1999). The temporal requirement for endothelin receptor-B signalling during neural crest development. Nature 402: 496-501.

Sieber-Blum M, Grim M, Hu YF, Szeder V (2004). Pluripotent neural crest stem cells in the adult hair follicle. Dev Dyn 231: 258-269.

Steingrimsson E, Copeland NG, Jenkins NA (2004). Melanocytes and the microphthalmia transcription factor network. Annu Rev Genet 38: 365-411.

Sucena E, Delon I, Jones I, Payre F, Stern DL (2003). Regulatory evolution of shavenbaby/ovo underlies multiple cases of morphological parallelism. Nature 424: 935-938.

Vance KW, Goding CR (2004). The transcription network regulating melanocyte development and melanoma. Pigment Cell Res 17: 318-325.

von Dassow G, Munro E (1999). Modularity in animal development and evolution: elements of a conceptual framework for EvoDevo. J Exp Zool 285: 307-325.

Wehrle-Haller B (2003). The role of Kit-ligand in melanocyte development and epidermal homeostasis. Pigment Cell Res 16: 287-296.

Yamamoto Y, Stock DW, Jeffery WR (2004). Hedgehog signalling controls eye degeneration in blind cavefish. Nature 431: 844-847. 